ARTICLE

\title{
The management of breast cancer-related lymphoedema
}

C M Marco, ${ }^{1}$ RN, MPhil MCH, Certified Lymphoedema Therapist (Norton School of Lymphatic Therapy, USA);

R Pillay, ${ }^{2}$ BSc OT, Certified Lymphoedema Therapist (Norton School of Lymphatic Therapy, USA);

C Schoonheim, ${ }^{3}$ BSc PT, Certified Lymphoedema Therapist (Norton School of Lymphatic Therapy, USA)

${ }^{1}$ Private Practice, Claremont, South Africa

${ }^{2}$ Department of Occupational Therapy, Groote Schuur Hospital, Cape Town, South Africa

Private Practice, Hout Bay, South Africa

Corresponding author: C M Marco (cmarco@telkomsa.net)

Lymphoedema is a chronic debilitating condition characterised by an accumulation of protein-rich fluid in interstitial spaces due to insufficient functioning of the lymphatic system. The condition may be referred to as primary (congenital malformation) or secondary (damage to the lymphatic system) lymphoedema. Lymphoedema is currently incurable, but can be alleviated with appropriate treatment. However, if ignored, it can progress and become difficult to manage.

S Afr Med J 2014;104(5):382. DOI:10.7196/SAMJ.8251

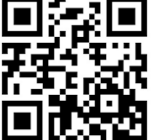

\section{Definition}

Breast cancer-related lymphoedema (BCRL) is a secondary form of lymphoedema and a major complication of post-breast cancer surgery and radiotherapy. It occurs when the network of lymphatic vessels and lymph nodes is damaged by disease, surgery or radiotherapy. The lymph, a protein-rich fluid, then accumulates in the tissue spaces, which is clinically manifested by swelling of the upper limb on the affected side. Sometimes the chest and breast areas may also be affected. ${ }^{[1,2]}$

\section{Classification and symptoms}

Lymphoedema can be classified into three stages:

- Stage 1 - subjective feeling of heaviness with or without visible swelling

- Stage 2 - visible swelling with pitting and perhaps numbness, heaviness, and pain

- Stage 3 - swelling, no pitting, fibrosis, skin changes, heaviness, numbness and pain.

If BCRL is not detected and treated in its early stages, it becomes chronic and debilitating with severe physical, psychosocial and economic implications for the breast cancer survivor's quality of life. ${ }^{[2,3]}$ The physiological factors include recurrent infections, limited movement of the limb due to pain, numbness and tenderness. The psychosocial factors include social isolation, anxiety, depression, and poor self- or body image. The economic factors include loss of employment and higher medical costs. ${ }^{[4-8]}$

\section{Risk factors}

After surgery and radiotherapy all breast cancer survivors are at risk of developing BCRL despite new surgical techniques to reduce its incidence. ${ }^{[1,7-10]}$ The lifetime risk of BCRL among breast cancer survivors varies from $33 \%$ to $44 \%$ and $7 \%$ to $44 \% \cdot{ }^{[3,9]}$ In addition, if lymphoedema is left untreated, it may cause recurrent infections in the affected arm, or a generalised systemic infection that may lead to death. ${ }^{[1-3,11]}$
Lymphoedema most commonly occurs in the first three years after breast cancer diagnosis and treatment; however, it may occur at any stage during the life of a breast cancer survivor. ${ }^{[8,10,11]}$

\section{Treatment/management}

There is currently no cure for BCRL, but there are international best practice guidelines to prevent or reduce its severity. ${ }^{[1]}$ The guidelines recommend complete decongestive therapy (CDT), which includes gentle massaging known as manual lymph drainage, use of a multilayered compression bandage to the affected limb, exercise, and skin/nail care. CDT facilitates functioning of the impaired lymphatic system and the eventual reduction of lymph volume in the affected upper limb.

Despite the presence of lymphoedema during or after breast cancer surgery, radiotherapy and lymph node dissection, its incidence and prevalence may be reduced in most cases if appropriate interventions are applied.

\section{International best practice consensus for lymphoedema management}

The international recommended treatment of BCRL is CDT, which is implemented in most economically developed countries in Europe, the USA, certain Asian countries and Australia. ${ }^{[1,2]}$

Various research studies have found that BCRL can develop at any time after breast cancer diagnosis and treatment. Therefore, it is recommended that preoperative and regular postoperative assessments of the upper limbs should be done to detect BCRL early. This, and immediate intervention, reduces the impact of BCRL on the quality of life of the affected breast cancer survivor. ${ }^{[1,3,8]}$

\section{Complete decongestive therapy}

$\mathrm{CDT}$, as the recommended gold standard, involves an initial intensive phase lasting 2 - 4 weeks, followed by the maintenance or transition phase described below. ${ }^{[1]}$

\section{Manual lymph drainage}

Manual lymph drainage (MLD) is a gentle massage technique comprising a series of strokes applied directly to the skin. This 
involves manual manipulation or 'soft tissue mobilisation' at a superficial and/or deep level of the lymphatic system and aims to move fluid away from the congested areas by increasing the activity of normal lymph vessels and bypassing ineffective or obliterated ducts. A typical session will involve drainage of the neck, trunk and involved extremity for $40-60$ minutes. It is recommended that MLD be performed daily during the intensive phase. Before embarking on MLD, all possible contraindications must be ruled out, including cellulitis, congestive heart failure, renal failure, and deep vein thrombosis. Other contraindications may be more specific to the patient's condition and a thorough history is therefore essential. ${ }^{[1}$

MLD is a specialised therapeutic skill and, although there is a wealth of clinical opinion advocating MLD, there is little evidence to support its efficacy. MLD is only effective when used in conjunction with other components.

Simple lymph drainage (SLD) is a simplified version of MLD that patients and healthcarers can be taught to self-administer. This can be performed for $10-20$ minutes daily. SLD can be conducted and taught by practitioners with appropriate training. ${ }^{[1]}$

\section{Multilayered lymphoedema bandaging compression therapy}

This involves the use of multilayered lymphoedema bandaging (MLLB) and compression garments. Lymphoedema bandaging or wrapping is a multilayered system of various sizes of short stretch, non-elastic pure cotton bandages applied over cotton padding using uniform spacing and tension to create compression around the affected area. The purpose of bandaging following MLD is to reduce the return of lymph to the affected area and to enhance increased lymph flow. Foam is often used in the layering to reduce fibrosis and increase comfort. During the intensive phase it is recommended that bandages be changed daily for the first 7 days and then be reduced to 2 - 3 times per week, depending on the progress. Self-bandaging or bandaging by a caregiver is also recommended if there is limited access to a skilled therapist.

Once adequate reduction of the affected limb is obtained, compression garments are applied to maintain the reduction. Garments can be custom-made or ready to use (off the shelf). Persons living with lymphoedema often wear the garments during the day and alternate these with bandaging at night during the maintenance phase of the intervention. Compression garments are essential for long-term improvement and assist with patient compliance. They can also be used prophylactically to prevent the progression of lymphoedema.

Garments can be categorised according to fabric construction (i.e. circular or flat knit) and the pressures exerted (Classes I - IV). The compression hosiery standards vary between different countries. ${ }^{[1]}$

Assessment and measurement of garments require that the caregiver or healthcare practitioner has specialist training or access to a lymphoedema specialist. ${ }^{[1]}$

\section{Exercise}

Exercise through the use of muscle contraction and deep breathing increases lymphatic flow and is most effective in conjunction with short stretch bandaging or compression garments. Exercise programmes, differing in frequency and intensity, are indicated for individuals with lymphoedema and for those at risk. Exercise can be categorised as lymphoedema exercise (non-resistive active motion of the affected limb), stretching (reducing tightness/scarring), resistive exercise (progressive weight resistance - this type poses the greatest risk) and aerobic conditioning (improving cardiovascular fitness). It should be noted that exercise alone will not reduce limb volume effectively and that any programme should be carefully explained and tailored to the individual's needs. ${ }^{[1]}$

\section{Skin care of the affected limb}

Maintenance of skin integrity and careful management of dermatological problems are important to minimise the risk of infection. The principles of skin care include: $:^{[1]}$

- Daily washing with a pH-neutral soap, natural soap or soap substitute and drying thoroughly.

- Ensuring that skin folds, if present, are clean and dry.

- Monitoring affected and unaffected skin for cuts, abrasions or insect bites, paying particular attention to areas affected by sensory neuropathy.

- Application of emollients.

- Avoidance of scented products, particularly in hot climates. Vegetable-based products are preferable to those containing petrolatum or mineral oils.

\section{Self-management}

Self-management is highly dependent on compliance and the individual's resources and support system. Self-management by the individual/caregiver involves education and training on massaging (SLD), bandaging, exercising and skin/nail care. As lymphoedema is a chronic condition, those affected by it have to master life-long management. Consistency is essential during self-management and therapeutic support is offered intermittently. ${ }^{[1]}$

\section{Challenges facing the management of} BCRL in South Africa

There are no official BCRL guidelines or protocols in the South African healthcare system. A skills gap exists in terms of the number of lymphoedema therapists in the public and private healthcare systems. Therefore, an accessible and effective service for those with the condition and at risk is lacking.

According to the newly established Lymphoedema Association of South Africa (LAOSA), there are approximately 67 lymphoedema therapists in South Africa. Fewer than $10 \%$ of them are currently working in the public sector. With the increased incidence of breast cancer and longer survival rates ( $>5$ years), the demand for lymphoedema treatment is steadily growing. Awareness of the condition is probably the major stumbling block in the healthcare system. The number of trained therapists cannot support or sustain a service according to the recommended gold standard method. Private lymphoedema therapists outnumber those in the public sector, where the majority of patients seek help. Budgetary constraints in terms of sourcing bandages and garments also hinder the implementation of gold standard management. Additionally, the socioeconomic status of patients makes it difficult to attend therapeutic sessions as often as recommended owing to financial hardship. Private patients receive little support from medical aids, some of which are only now starting to consider covering CDT treatment and only by certain categories of professionals.

\section{Conclusion}

The recommended international best practice guidelines cannot be implemented at all levels of care in the South African public and private healthcare sectors owing to the paucity of healthcare providers with the requisite skills to manage BCRL. In addition, the compression garments and bandages are very costly and rarely available in the public healthcare sector. Most medical aids do not provide full reimbursement for the cost of these commodities. Against this backdrop BCRL referral and management guidelines and other recommendations are proposed for South Africa (Tables 1 and 2). 


\section{Recommendations}

1. All newly diagnosed patients and those with BCRL symptoms should be referred appropriately.

Table 1. Referral pathway guideline within and from the various levels of care

\begin{tabular}{ll}
\hline Level of care & Guideline \\
\hline Level 3/level 2: tertiary/secondary hospital (public health) & $\begin{array}{l}\text { All newly diagnosed patients to hospital-based lymphoedema service for BCRL } \\
\text { information and pre-treatment measurements of limbs } \\
\text { Patients with BCRL symptoms to lymphoedema service for assessment and } \\
\text { treatment plan }\end{array}$ \\
$\begin{array}{ll}\text { Pevel 1: primary level clinics, community healthcare centres } \\
\text { BCRL = breast cancer-related lymphoedema. }\end{array}$ & $\begin{array}{l}\text { assessment and management and to home-based carer/rehabilitation careworker } \\
\text { to provide support within the community }\end{array}$ \\
&
\end{tabular}

2. Patients who present with various stages of BCRL should be managed appropriately.

Table 2. Staging and management of breast cancer-related lymphoedema

\begin{tabular}{ll}
\hline Stage of lymphoedema & Treatment/management \\
\hline Stage1: feeling of heaviness only, accompanied by slight swelling & $\begin{array}{l}\text { Symptoms relieved by elevation of the limb, exercise, compression garment if } \\
\text { slight swelling persists }\end{array}$ \\
$\begin{array}{l}\text { Stage 2: visible swelling and pitting, with/without hardening of } \\
\text { the skin, slight change in skin texture }\end{array}$ & $\begin{array}{l}\text { Encourage exercise of limb, manual lymph drainage/simple lymph drainage, } \\
\text { skin care, exercise, use of multilayer compression bandaging }\end{array}$ \\
Stage 3: visible swelling, no pitting, fibrosis, skin changes & $\begin{array}{l}\text { Encourage exercise of limb, manual lymph drainage/simple lymph drainage, } \\
\text { skin care, exercise, multilayered lymphoedema bandaging }\end{array}$
\end{tabular}

Managing BCRL is very intense and could become cumbersome for the therapist, caregiver and affected person. It is therefore important to keep the patient motivated. The treatment plan should be unique to each individual.

3. National Department of Health $(\mathrm{NDoH})$ to facilitate development of a breast health-cancer policy that includes BCRL management guidelines.

4. $\mathrm{NDoH}$ to facilitate integration of BCRL management guidelines into the chronic diseases directorate policy, as lymphoedema is a chronic condition.

5. BCRL epidemiology and management to be integrated into training curricula of healthcare professionals and rehabilitation careworkers (RCWs)/home-based carers (HBCs).

6. Research to be conducted to inform the development of BCRL treatment protocols in South Africa.

7. An integrated team approach, which includes oncologists, surgeons, nurses, doctors, physiotherapists, occupational therapists, social workers, RCWs/HBCs and, most importantly, the individual at risk of/experiencing BCRL.

8. The development of lymphoedema packages of care/treatment guidelines for different levels of care suited to South Africa.

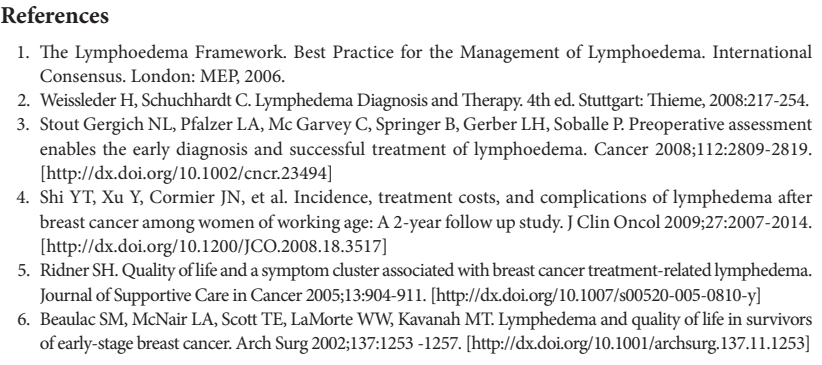

1. The Lymphoedema Framework. Best Practice for the Management of Lymphoedema. International Consensus. London: MEP, 2006.

2. Weissleder H, Schuchhardt C. Lymphedema Diagnosis and Therapy. 4th ed. Stuttgart: Thieme, 2008:217-254. 3. Stout Gergich NL, Pfalzer LA, Mc Garvey C, Springer B, Gerber LH, Soballe P. Preoperative assessment enables the early diagnosis and successful treatment of lymphoedema. Cancer 2008;112:2809-2819. [http://dx.doi.org/10.1002/cncr.23494]

4. Shi YT, Xu Y, Cormier JN, et al. Incidence, treatment costs, and complications of lymphedema after breast cancer among women of working age: A 2-year follow up study. J Clin Oncol 2009;27:2007-2014. [http://dx.doi.org/10.1200/JCO.2008.18.3517]

5. Ridner SH. Quality of life and a symptom cluster associated with breast cancer treatment-related lymphedema. Journal of Supportive Care in Cancer 2005;13:904-911. [http://dx.doi.org/10.1007/s00520-005-0810-y]

6. Beaulac SM, McNair LA, Scott TE, LaMorte WW, Kavanah MT. Lymphedema and quality of life in survivors of early-stage breast cancer. Arch Surg 2002;137:1253 -1257. [http://dx.doi.org/10.1001/archsurg.137.11.1253]

7. Petrek JA, Senie RT, Peters M, Rosen PP. Lymphedema in a cohort of breast carcinom survivors 20 years after diagnosis. Cancer 2001;92:1368-1377. [http://dx.doi.org/10.1002/10970142(20010915)92:6<1368::AID-CNCR1459>3.0.CO;2-9]

8. Hayes SC, Janda M, Cornish B, Battistutta D, Newman B. Lymphedema after breast cancer: Incidence, risk factors and effect on upper body function. J Clin Oncol 2008;26:3536-3542. [http://dx.doi. org/10.1200/JCO.2007.14.4899]

9. Meek AG. Breast radiotherapy and lymphedema. Cancer 1998;83:2788-2797. [http://dx.doi.org/10.1002/ (SICI) 1097-0142(19981215)83:12B +<2788::AID-CNCR27>3.0.CO;2-I]

10. Norman SA, Localio R, Potashnik SL, et al. Lymphoedema in breast cancer survivors: Incidence, degree, time course, treatment and symptoms. J Clin Oncol 2009;27:390-397. [http://dx.doi.org/10.1200/ JCO.2008.17.9291]

11. Clark B, Sitza J, Harlow W. Incidence and risk of arm oedema following treatment for breast cancer: A three year follow-up study. QJM 2005;98:343-348. 